\title{
AGE INCIDENCE OF DYSMENORRHOEA
}

\author{
BY \\ C. R. LOWE and R. L. FERGUSON \\ From the Department of Social Medicine, University of Birmingham, and the \\ Medical Department, Cadbury Bros. Ltd., Bournville
}

Since dysmenorrhoea refers to an ill-defined group of symptoms, it is not surprising that estimates of its incidence in women of child-bearing age range from 3 to 90 per cent. Most women experience some abdominal discomfort either immediately before or during menstruation, and the degree of discomfort called dysmenorrhoea will depend upon the individual patient's tolerance of pain, the nature of the population examined, and the investigator's criteria and interests.

If dysmenorrhoea is defined as a degree of menstrual pain sufficient to interfere with a woman's normal mode of life, estimates of incidence range from about 3 to 17 per cent. Most of these estimates are the result of questioning university students (Bell and Parsons, 1931; Cunningham, 1934), hospital patients (Emge, 1933; Bertling, 1948), hospital nurses (Larkin, 1941), or high-school girls (Goldwasser, 1938), and are by no means representative of the general female population. Several investigations have been concerned with industrial workers (Ewing, 1931; Lakeman, 1933), but no attempt appears to have been made to relate incidence to age or to marital status.

\section{DATA}

In 1927, Cadbury Brothers Ltd., Bournville, established a retiring room for women workers. This room is equipped with six camp beds, and hot-water bottles and analgesics are available under the supervision of a first-aid worker. Over the six 4-weekly periods from January 1 to June 17,1950 , a record was kept of all workers using the room who complained specifically of menstrual pain. The total works population by age and marital status for each of the months under consideration was known, and the note which follows relates this information to data from the retiring-room records.

Before proceeding, however, we must first draw attention to certain limitations of the data:

(1) Inclusion in the series depends upon each patient's decision to use the retiring room.

(2) The proportion of women who stay away from work because of dysmenorrhoea is unknown. There is reason to believe that the number is small, however, as, during the period under consideration, less than 2 per cent. of the female population had single or half-day absences from work for 3 consecutive months (the corresponding figure for male employees was about 0.2 per cent.).

(3) A few women with intermenstrual intervals of less than 4 weeks may have used the room twice within any one month, and are therefore represented twice within that month. Two visits on the same day by the same woman are counted as a single visit and the 
duration of the visit is recorded as the total time spent in the retiring room during that day.

(4) Although the majority of women using the retiring room do so fairly regularly, by no means all of them report sick every month.

There are, therefore, obvious objections to using the data as a measure of the incidence

TABLE I

Percentage of Single Women attending Retiring Room because of Dysmenorrhoea

\begin{tabular}{|c|c|c|c|c|c|c|c|}
\hline \multirow{2}{*}{4 weeks ending } & \multicolumn{6}{|c|}{ Age Group (years) } & \multirow{2}{*}{ All Age } \\
\hline & $15-19$ & $20-24$ & $25-29$ & $30-34$ & $35-39$ & $40-44$ & \\
\hline January 28 & $\begin{array}{l}6 \cdot 8 \\
(892)^{*}\end{array}$ & $\begin{array}{l}13 \cdot 5 \\
(495)\end{array}$ & $\begin{array}{c}5 \cdot 3 \\
(282)\end{array}$ & $\begin{array}{c}7 \cdot 5 \\
(133)\end{array}$ & $\begin{array}{c}0.9 \\
(108)\end{array}$ & $\begin{array}{c}2 \cdot 7 \\
(149)\end{array}$ & $\begin{array}{c}7 \cdot 7 \\
(2,059)\end{array}$ \\
\hline February 25 & $\begin{array}{c}6 \cdot 7 \\
(859)\end{array}$ & $\begin{array}{l}10 \cdot 6 \\
(499)\end{array}$ & $\begin{array}{c}7 \cdot 1 \\
(281)\end{array}$ & $\begin{array}{c}2 \cdot 1 \\
(145)\end{array}$ & $\begin{array}{c}0 \cdot 0 \\
(120)\end{array}$ & $\begin{array}{c}2 \cdot 0 \\
(152)\end{array}$ & $\begin{array}{c}6 \cdot 7 \\
(2,056)\end{array}$ \\
\hline March 25 & $\begin{array}{c}6 \cdot 3 \\
(829)\end{array}$ & $\begin{array}{l}11 \cdot 2 \\
(463)\end{array}$ & $\begin{array}{c}6 \cdot 8 \\
(280)\end{array}$ & $\begin{array}{c}2 \cdot 3 \\
(132)\end{array}$ & $\begin{array}{c}0 \cdot 0 \\
(107)\end{array}$ & $\begin{array}{c}0.6 \\
(155)\end{array}$ & $\begin{array}{c}6 \cdot 5 \\
(1,966)\end{array}$ \\
\hline April 22 & $\begin{array}{c}5 \cdot 5 \\
(850)\end{array}$ & $\begin{array}{c}8 \cdot 9 \\
(473)\end{array}$ & $\begin{array}{c}2 \cdot 5 \\
(276)\end{array}$ & $\begin{array}{c}4 \cdot 6 \\
(130)\end{array}$ & $\begin{array}{c}0.9 \\
(110)\end{array}$ & $\begin{array}{c}1 \cdot 3 \\
(153)\end{array}$ & $\begin{array}{c}5 \cdot 3 \\
(1,992)\end{array}$ \\
\hline May 20 & $\begin{array}{c}7 \cdot 0 \\
(828)\end{array}$ & $\begin{array}{l}10 \cdot 6 \\
(472)\end{array}$ & $\begin{array}{l}10 \cdot 8 \\
(279)\end{array}$ & $\begin{array}{c}0 \cdot 8 \\
(124)\end{array}$ & $\begin{array}{c}0 \cdot 0 \\
(114)\end{array}$ & $\begin{array}{c}2 \cdot 0 \\
(150)\end{array}$ & $\begin{array}{c}7 \cdot 2 \\
(1,967)\end{array}$ \\
\hline June 17 & $\begin{array}{c}4 \cdot 2 \\
(826)\end{array}$ & $\begin{array}{c}6 \cdot 4 \\
(470)\end{array}$ & $\begin{array}{c}4 \cdot 3 \\
(276)\end{array}$ & $\begin{array}{c}1 \cdot 6 \\
(129)\end{array}$ & $\begin{array}{c}0.9 \\
(111)\end{array}$ & $\begin{array}{c}0 \cdot 7 \\
(149)\end{array}$ & $\begin{array}{c}4 \cdot 1 \\
(1,961)\end{array}$ \\
\hline
\end{tabular}

* Number of related employees in brackets.

TABLE II

Percentage of Married Women attending Retiring Room because of Dysmenorrhoea

\begin{tabular}{|c|c|c|c|c|c|c|c|}
\hline \multirow{2}{*}{4 weeks ending } & \multicolumn{6}{|c|}{ Age Group (years) } & \multirow{2}{*}{ All Ages } \\
\hline & $15-19$ & $20-24$ & $25-29$ & $30-34$ & $35-39$ & $40-44$ & \\
\hline January 28 & $\begin{array}{l}0 \cdot 0 \\
(8)^{*}\end{array}$ & $\begin{array}{c}9 \cdot 6 \\
(178)\end{array}$ & $\begin{array}{c}2 \cdot 9 \\
(277)\end{array}$ & $\begin{array}{c}1 \cdot 5 \\
(202)\end{array}$ & $\begin{array}{c}1 \cdot 3 \\
(235)\end{array}$ & $\begin{array}{c}0 \cdot 5 \\
(219)\end{array}$ & $\begin{array}{c}2 \cdot 9 \\
(1,119)\end{array}$ \\
\hline February 25 & $\begin{array}{l}0 \cdot 0 \\
(7)\end{array}$ & $\begin{array}{c}6 \cdot 5 \\
(170)\end{array}$ & $\begin{array}{c}3 \cdot 0 \\
(263)\end{array}$ & $\begin{array}{c}2 \cdot 7 \\
(184)\end{array}$ & $\begin{array}{c}1 \cdot 0 \\
(208)\end{array}$ & $\begin{array}{c}0.9 \\
(216)\end{array}$ & $\begin{array}{c}2 \cdot 7 \\
(1,048)\end{array}$ \\
\hline March 25 & $\begin{array}{l}0.0 \\
(9)\end{array}$ & $\begin{array}{c}6 \cdot 0 \\
(150)\end{array}$ & $\begin{array}{c}4 \cdot 5 \\
(200)\end{array}$ & $\begin{array}{c}2 \cdot 5 \\
(119)\end{array}$ & $\begin{array}{c}1 \cdot 4 \\
(144)\end{array}$ & $\begin{array}{c}0 \cdot 0 \\
(150)\end{array}$ & $\begin{array}{c}3 \cdot 0 \\
(772)\end{array}$ \\
\hline April 22 & $\begin{array}{l}0 \cdot 0 \\
(10)\end{array}$ & $\begin{array}{c}3 \cdot 8 \\
(156)\end{array}$ & $\begin{array}{c}3 \cdot 6 \\
(197)\end{array}$ & $\begin{array}{c}4 \cdot 3 \\
(117)\end{array}$ & $\begin{array}{c}0 \cdot 7 \\
(144)\end{array}$ & $\begin{array}{c}2 \cdot 0 \\
(151)\end{array}$ & $\begin{array}{c}2 \cdot 8 \\
(775)\end{array}$ \\
\hline May 20 & $\begin{array}{l}0 \cdot 0 \\
(8)\end{array}$ & $\begin{array}{c}5 \cdot 9 \\
(152)\end{array}$ & $\begin{array}{c}9 \cdot 0 \\
(211)\end{array}$ & $\begin{array}{c}2 \cdot 0 \\
(153)\end{array}$ & $\begin{array}{c}2 \cdot 2 \\
(180)\end{array}$ & $\begin{array}{c}0 \cdot 5 \\
(190)\end{array}$ & $\begin{array}{c}4 \cdot 0 \\
(894)\end{array}$ \\
\hline June 17 & $\begin{array}{l}0 \cdot 0 \\
(9)\end{array}$ & $\begin{array}{c}3 \cdot 0 \\
(167)\end{array}$ & $\begin{array}{c}2 \cdot 0 \\
(253)\end{array}$ & $\begin{array}{c}0.0 \\
(202)\end{array}$ & $\begin{array}{c}0 \cdot 4 \\
(237)\end{array}$ & $\begin{array}{c}0 \cdot 0 \\
(225)\end{array}$ & $\begin{array}{c}1 \cdot 0 \\
(1,093)\end{array}$ \\
\hline
\end{tabular}

* Number of related employees in brackets. 
of dysmenorrhoea in the general population, but these objections are unlikely to invalidate any trend observed in relation to age or marital status.

\section{RESULTS}

Examined by age, the proportion of both single and married women using the retiring room because of dysmenorrhoea shows a fairly regular pattern at each of the six 4-weekly intervals (Tables I and II). Data collected over the 6 months are summarized in Table III (see also the Figure). The proportion of women using the retiring room is highest at 20-24 years $(9 \cdot 1$ per cent.) and decreases with advancing age. No married woman under 20 years used the room (the population at risk was very small). It is just possible that the comparatively low incidence of single girls $(6 \cdot 1$ per cent.) in the lowest age group may be due to an appreciable number not yet having reached the menarche, but although we have no direct information, this explanation is unlikely, as it is recognized that the mean age of the menarche is under 14 years and that more than 90 per cent. of girls have experienced their first menstrual loss by their

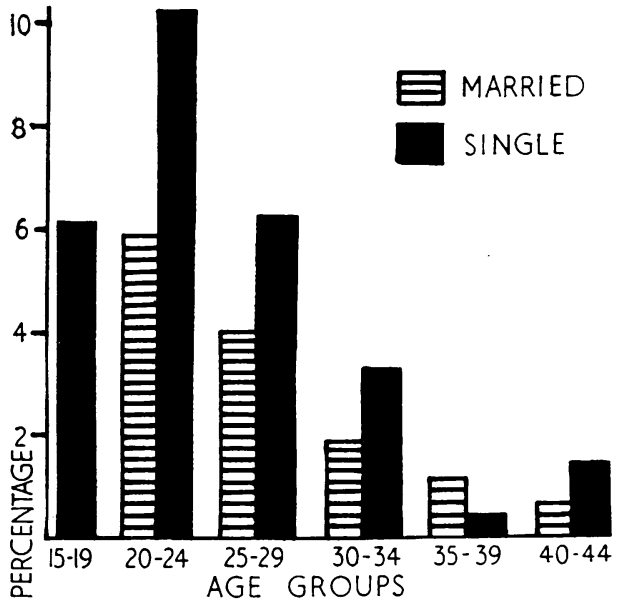

FIGURE.-Percentage of women attending retiring room because of dysmenorrhoea, by age groups. fifteenth birthday (Engle and Shelesnyack, 1934; Hogben and others, 1948). It is more likely that this low incidence in young women supports the statement commonly made in gynaecological textbooks that the menarche may be followed by several months or

TABLE III

Mean Monthly Percentage of Women attending Retiring RoOm because of Dysmenorrhoea

\begin{tabular}{|c|c|c|c|c|c|c|c|c|}
\hline \multirow{2}{*}{\multicolumn{2}{|c|}{ Marital Status }} & \multicolumn{6}{|c|}{ Age Group (years) } & \multirow{2}{*}{ All Ages } \\
\hline & & $15-19$ & $20-24$ & $25-29$ & $30-34$ & $35-39$ & $40-44$ & \\
\hline Single & . & $\begin{array}{c}6 \cdot 1 \\
(847) *\end{array}$ & $\begin{array}{l}10 \cdot 2 \\
(479)\end{array}$ & $\begin{array}{c}6 \cdot 2 \\
(279)\end{array}$ & $\begin{array}{c}3 \cdot 2 \\
(132)\end{array}$ & $\begin{array}{c}0.4 \\
(112)\end{array}$ & $\begin{array}{c}1 \cdot 5 \\
(151)\end{array}$ & $\begin{array}{c}6 \cdot 2 \\
(2,000)\end{array}$ \\
\hline Married & . & $\begin{array}{l}0 \cdot 0 \\
(9)\end{array}$ & $\begin{array}{c}5 \cdot 9 \\
(162)\end{array}$ & $\begin{array}{c}4 \cdot 0 \\
(233)\end{array}$ & $\begin{array}{c}1 \cdot 9 \\
(163)\end{array}$ & $\begin{array}{c}1 \cdot 1 \\
(181)\end{array}$ & $\begin{array}{c}0 \cdot 6 \\
(192)\end{array}$ & $\begin{array}{c}2 \cdot 7 \\
(950)\end{array}$ \\
\hline Total & . & $\begin{array}{c}6 \cdot 1 \\
(856)\end{array}$ & $\begin{array}{c}9 \cdot 1 \\
(641)\end{array}$ & $\begin{array}{c}5 \cdot 2 \\
(512)\end{array}$ & $\begin{array}{c}2 \cdot 5 \\
(295)\end{array}$ & $\begin{array}{c}0.9 \\
(303)\end{array}$ & $\begin{array}{c}1 \cdot 0 \\
(343)\end{array}$ & $\begin{array}{c}5 \cdot 1 \\
(2,950)\end{array}$ \\
\hline
\end{tabular}

* Mean monthly number of related employees in brackets. 
TABLE IV

Mean Time (min.) spent by Patients in Retiring Room

\begin{tabular}{|c|c|c|c|c|c|c|c|}
\hline \multirow{2}{*}{4 weeks ending } & \multicolumn{6}{|c|}{ Age Group (years) } & \multirow{2}{*}{ All Ages } \\
\hline & $15-19$ & $20-24$ & $25-29$ & $30-34$ & $35-39$ & $40-44$ & \\
\hline January 28 & $\begin{array}{l}29 \cdot 0 \\
(61)^{*}\end{array}$ & $\begin{array}{l}27 \cdot 7 \\
(84)\end{array}$ & $\begin{array}{l}28 \cdot 3 \\
(23)\end{array}$ & $\begin{array}{l}19 \cdot 2 \\
(13)\end{array}$ & $\begin{array}{c}18 \cdot 2 \\
(4)\end{array}$ & $\begin{array}{l}14 \cdot 0 \\
\cdot(5)\end{array}$ & $\begin{array}{l}27 \cdot 0 \\
(190)\end{array}$ \\
\hline February 25 & $\begin{array}{l}19 \cdot 7 \\
(58)\end{array}$ & $\begin{array}{l}23 \cdot 0 \\
(64)\end{array}$ & $\begin{array}{l}11 \cdot 9 \\
(28)\end{array}$ & $\begin{array}{c}11 \cdot 1 \\
(8)\end{array}$ & $\begin{array}{l}4 \cdot 0 \\
(2)\end{array}$ & $\begin{array}{l}3 \cdot 2 \\
(5)\end{array}$ & $\begin{array}{l}18 \cdot 5 \\
(165)\end{array}$ \\
\hline March 25 & $\begin{array}{l}23 \cdot 6 \\
(52)\end{array}$ & $\begin{array}{l}17 \cdot 1 \\
(61)\end{array}$ & $\begin{array}{l}19 \cdot 4 \\
(28)\end{array}$ & $\begin{array}{c}12 \cdot 5 \\
(6)\end{array}$ & $\begin{array}{l}3 \cdot 0 \\
(2)\end{array}$ & $\begin{array}{l}3 \cdot 0 \\
(1)\end{array}$ & $\begin{array}{l}19 \cdot 3 \\
(150)\end{array}$ \\
\hline April 22 & $\begin{array}{l}20 \cdot 2 \\
(47)\end{array}$ & $\begin{array}{c}20 \cdot 3 \\
(48)\end{array}$ & $\begin{array}{l}18 \cdot 8 \\
(14)\end{array}$ & $\begin{array}{l}17 \cdot 8 \\
(11)\end{array}$ & $\begin{array}{c}31 \cdot 0 \\
(2)\end{array}$ & $\begin{array}{l}2 \cdot 5 \\
(5)\end{array}$ & $\begin{array}{l}19 \cdot 3 \\
(127)\end{array}$ \\
\hline May 20 & $\begin{array}{l}26 \cdot 1 \\
(58)\end{array}$ & $\begin{array}{l}22 \cdot 1 \\
(59)\end{array}$ & $\begin{array}{l}21 \cdot 0 \\
(49)\end{array}$ & $\begin{array}{l}2 \cdot 5 \\
(4)\end{array}$ & $\begin{array}{l}2 \cdot 5 \\
(4)\end{array}$ & $\begin{array}{l}2 \cdot 7 \\
(4)\end{array}$ & $\begin{array}{l}21 \cdot 8 \\
(178)\end{array}$ \\
\hline June 17 & $\begin{array}{c}31 \cdot 4 \\
(35)\end{array}$ & $\begin{array}{l}23 \cdot 6 \\
(35)\end{array}$ & $\begin{array}{l}11 \cdot 7 \\
(17)\end{array}$ & $\begin{array}{c}31 \cdot 0 \\
(2)\end{array}$ & $\begin{array}{l}3 \cdot 0 \\
(2)\end{array}$ & $\begin{array}{l}77 \cdot 0 \\
\text { (1) }\end{array}$ & $\begin{array}{l}24 \cdot 7 \\
(92)\end{array}$ \\
\hline Monthly Mean & $\begin{array}{l}24 \cdot 7 \\
(52)\end{array}$ & $\begin{array}{l}22 \cdot 7 \\
(59)\end{array}$ & $\begin{array}{l}18 \cdot 9 \\
(26)\end{array}$ & $\begin{array}{c}15 \cdot 5 \\
(7)\end{array}$ & $\begin{array}{c}10 \cdot 3 \\
(3)\end{array}$ & $\begin{array}{l}9 \cdot 0 \\
(3)\end{array}$ & $\begin{array}{l}21 \cdot 8 \\
(150)\end{array}$ \\
\hline
\end{tabular}

* Number of patients in brackets.

TABLE $\mathrm{V}$

Percentage of Patients with Dysmenorrhoea who complained of Severe Backache

\begin{tabular}{|c|c|c|c|c|c|c|c|c|c|}
\hline \multirow{2}{*}{\multicolumn{5}{|c|}{4 weeks ending }} & \multicolumn{4}{|c|}{ Age Group (years) } & \multirow{3}{*}{$\begin{array}{c}\text { All Ages } \\
\begin{array}{c}15 \cdot 3 \\
(190)\end{array}\end{array}$} \\
\hline & & & & & \multirow{2}{*}{$\begin{array}{c}15-19 \\
11 \cdot 5 \\
(61)^{*}\end{array}$} & \multirow{2}{*}{$\begin{array}{c}20-24 \\
16 \cdot 7 \\
(84)\end{array}$} & \multirow{2}{*}{$\begin{array}{c}25-29 \\
13 \cdot 0 \\
(23)\end{array}$} & \multirow{2}{*}{$\begin{array}{r}+30-44 \\
22 \cdot 7 \\
(22)\end{array}$} & \\
\hline January 28 & . & . & . & . & & & & & \\
\hline February 25 & . & . & $\cdots$ & . & $\begin{array}{l}5 \cdot 2 \\
(58)\end{array}$ & $\begin{array}{l}17 \cdot 2 \\
(64)\end{array}$ & $\begin{array}{l}17 \cdot 9 \\
(28)\end{array}$ & $\begin{array}{l}26 \cdot 7 \\
(15)\end{array}$ & $\begin{array}{l}13 \cdot 9 \\
(165)\end{array}$ \\
\hline March 25 & . & . & $\cdots$ & . & $\begin{array}{l}3 \cdot 8 \\
(52)\end{array}$ & $\begin{array}{l}8 \cdot 2 \\
(61)\end{array}$ & $\begin{array}{l}10 \cdot 7 \\
(28)\end{array}$ & $\begin{array}{c}44 \cdot 4 \\
(9)\end{array}$ & $\begin{array}{c}9 \cdot 3 \\
(150)\end{array}$ \\
\hline April 22 & . & $\ldots$ & $\cdots$ & $\ldots$ & $\begin{array}{l}8 \cdot 5 \\
(47)\end{array}$ & $\begin{array}{l}14 \cdot 6 \\
(48)\end{array}$ & $\begin{array}{l}7 \cdot 1 \\
(14)\end{array}$ & $\begin{array}{l}22 \cdot 2 \\
(18)\end{array}$ & $\begin{array}{l}12 \cdot 6 \\
(127)\end{array}$ \\
\hline May 20 & $\cdots$ & 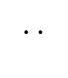 & . & . & $\begin{array}{l}5 \cdot 2 \\
(58)\end{array}$ & $\begin{array}{l}5 \cdot 1 \\
(59)\end{array}$ & $\begin{array}{c}20 \cdot 4 \\
(49)\end{array}$ & $\begin{array}{c}25 \cdot 0 \\
(12)\end{array}$ & $\begin{array}{l}10 \cdot 7 \\
(178)\end{array}$ \\
\hline June 17 & $\ldots$ & $\ldots$ & $\ldots$ & . & $\begin{array}{l}8 \cdot 6 \\
(35)\end{array}$ & $\begin{array}{l}14 \cdot 3 \\
(35)\end{array}$ & $\begin{array}{c}29 \cdot 4 \\
(17)\end{array}$ & $\begin{array}{l}0 \cdot 0 \\
(5)\end{array}$ & $\begin{array}{l}14 \cdot 1 \\
(92)\end{array}$ \\
\hline \multirow{2}{*}{\multicolumn{2}{|c|}{ Monthly Mean }} & \multicolumn{3}{|c|}{ All Patients } & $\begin{array}{l}7 \cdot 1 \\
(52)\end{array}$ & $\begin{array}{l}12 \cdot 8 \\
(59)\end{array}$ & $\begin{array}{l}17 \cdot 0 \\
(26)\end{array}$ & $\begin{array}{l}24 \cdot 7 \\
(13)\end{array}$ & $\begin{array}{l}12 \cdot 6 \\
(150)\end{array}$ \\
\hline & & \multicolumn{3}{|c|}{ Single Patients } & $\begin{array}{l}7 \cdot 1 \\
(52)\end{array}$ & $\begin{array}{l}12 \cdot 2 \\
(49)\end{array}$ & $\begin{array}{l}18 \cdot 4 \\
(17)\end{array}$ & $\begin{array}{c}23 \cdot 8 \\
(7)\end{array}$ & $\begin{array}{l}11 \cdot 6 \\
(125)\end{array}$ \\
\hline
\end{tabular}

* Number of patients in brackets. $\dagger$ Numbers do not justify subdivision of the age group 30-44. 
even years of painless menstruation. The average time spent by patients in the retiring room decreases regularly and rapidly with advancing age (Table IV). This suggests that, although the incidence of dysmenorrhoea under 20 years of age is low, it is likely to be more severe then than at any later age.

With the exception of the age group 35-39 years, in which the numbers are very small, a higher proportion of single than married women use the retiring room at all ages. It is evident that marriage (and possibly parity) has an appreciable effect in diminishing the severity of menstrual pain, but the marked decrease in incidence with advancing age is not fully explained either by marital status or parity, since single women show a similar age trend. Table $\mathrm{V}$ shows that the proportion of women using the retiring room who complain of severe backache during menstruation increases rapidly with age and that this also bears little relation to marital status.

We have pleasure in acknowledging our thanks to the staff of the retiring room for their co-operation, and to Miss M. Flear of Cadbury's Statistical Department for help in tabulating the data.

\section{REFERENCES}

Bell, M., and Parsons, E. (1931). Med. Wom. J., 38, 31.

Bertling, M. H. (1948). Amer. J. Obstet. Gynec., 56, 733.

Cunningham, R. L. (1934). West. J. Surg., 42, 274.

Emge, L. A. (1933). Calif. west. Med., 39, 380.

Engle, E. T., and Shelesnyak, M. C. (1934). Hum. Biol., 6, 431.

Ewing, R. E. (1931). J. industr. Hyg., 13, 244.

Goldwasser, M. (1938). Calif. west. Med., 48, 418.

Hogben, H., Waterhouse, J. A. H., and Hogben, L. (1948). British Journal of Social Medicine, 2, 29.

Lakeman, M. R. (1933). New Engl. J. Med., 209, 237.

Larkin, W. J. (1941). Penn. med. J., 44, 994. 